Das Gesamtwerk wird von keinem Leser ohne Gewinn aus der Hand gelegt werden.

F. V. Hess.

Himmelsglobus aus Modelliernetzen. Mit einer Anleitung "Der Sternenhimmel", 2. Auflage. Von A. Höfler. Teubner, 1913.

Dieses Modell des Sternenhimmels, das jeder dreizehnjährige Schüler aus dem gegebenen Material anfertigen kann, ist als ein vorzügliches Lehrmittel zu empfehlen. Die südliche Kappe der Kugel fehlt, dadurch wird das Hineinsehen in die Hohlkugel ermögiicht, die Sternbilder sind auf der Außenseite markiert, werden einzeln, nachdem man sie am Himmel aufgefunden hat, durchstochen und erscheinen dadurch, im Gegensatz zu ihrer Darstellung auf den gewöhnlichen Himmelsgloben, in derselben Stellung, in der sie beobachtet werden. Der Globus ist auch in fertigem Zustand durch den Verlag um mäßigen Preis zu beziehen.

$F$.

\title{
Didaktik der Himmelskunde und astronomischen Geographie.
} Von A. Höfler. XII + 414 Seiten. Teubner, 1913.

Die österreichischen Lehrpläne von 1908 haben den Unterricht in der Himmelskunde and astronomischen Geographie über den Lehrstoff aller Klassen etwa so verteilt, wie der Verfasser es seit langem auf Grund vielfacher Bemühungen und Erfahrungen in seiner Unterrichtspraxis gefordert hatte. Die vorliegende „Didaktik" kann zum Teil als eine Sammlung von Vorschlägen für die Durchführung dieser Lehrpläne betrachtet werden - Vorschläge, die in der Tat wegen der Kürze des offiziellen Lehrplantextes sehr zu begrüßen sind. Die Dispositionsvorschläge des Verfassers seien hier in Kürze zusammengefaßt.

1. Schuljahr: Beobachtung der täglichen Sonnenbahn und des höchsten Sonnenstandes, Schatten. Schließliches Ergebnis: Die jährliche Schraubenbahn. 2. Jahr: Verschiedene Stellung der Schraubenbahn in verschiedenen Gegenden ("Landschaftsbilder"); daraus, daß die Bahn der Sonne für den nach Süden Wandernden sich nicht merklich erweitert, wird auf deren große Entfernung, auf den Parallelismus aller die Erde treffenden Strahlen, somit auf die verschiedene Neigung des Horizontes gegen die Strahlen an verschiedenen Erdorten geschlossen. Die Proportionalität zwischen der Aufrichtung der Sonnenbahn zu dem nach Süden zurückgelegten Wege ergibt die Kreisgestalt des Nord-Südweges. Analog ergibt sich die des Ost-Westweges, damit die Kugelgestalt der Erde und - nach der Messung des Eratosthenes - die Größe ihres Durchmessers. Erweiterung der Nord - Südlinie zur Meridianlinie; Meridianebene, Erdachse, Pole, Globus. 3. Jahr: Gnomon. Orientierung am Fixsternhimmel, seine tägliche Bewegung. Beobachtung des Mondes, seiner Phasen und Stellungen gegen die Erde und gegen die Fixsterne. Tierkreisgürtel. Himmelsglobus. Sonnenstellangen im Tierkreis, Ekliptik. Erklärung der Mondphasen, Angabe von Distanzen. 4. Jahr: Heliozentrische Erklärung der bisher beobachteten Erscheinungen. Für alle Klassen der Oberstufe werden mathematische Aufgaben zur Astronomie, zumeist nach Schülkes Aufgabensammlung, vorgeschlagen, für den Physikunterricht der VII. und VIII. Klasse wird die Einschaltung der mechanisehen Erklärung astronomischer Erscheinungen in den Physikunterricht nach des Verfassers "Lehrbuch der Physik" empfohlen. 